\section{Le Grand Prix Inserm 2020 \\ a honoré \\ Dominique Costagliola}

Marcel Goldberg

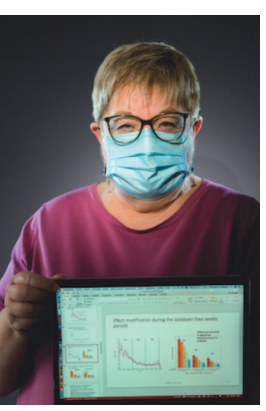

Unité Cohortes épidémiologiques en populationInserm/Université de Paris/Université Paris Saclay/ UVSQ, 16 avenue Paul-Vaillant-Couturier, F-94800 Villejuif, France. marcel.goldberg@inserm.fr

active, en particulier chez les hommes qui ont des relations sexuelles avec les hommes. Les modèles de diffusion de l'épidémie montrent en effet que le paramètre clé pour le contrôle de l'épidémie est le délai entre l'infection et le fait d'avoir une charge virale contrôlée, synonyme d'absence de risque de transmission. Ces modèles montrent qu'il faudrait que $90 \%$ des personnes infectées soient traitées dans l'année qui suit leur infection pour espérer contrôler l'épidémie grâce au traitement. Or, en France, le délai entre infection et diagnostic est alors en médiane de 3 ans et 2 mois, indiquant ainsi que, avec cette durée de dépistage, il ne sera pas possible de contrôler l'épidémie par le traitement sans, en parallèle, prévenir l'acquisition de nouvelles infections. C'est pourquoi, la prophylaxie préexposition (ou PrEP) chez les personnes à haut risque d'infection est une avancée majeure et Dominique Costagliola en sera une actrice majeure. Elle préside le comité indépendant de l'essai Ipergay (qui sera interrompu précocement car il montre rapidement son efficacité : $86 \%$ de réduction du risque d'acquisition de l'infection). Elle coordonne avec Jean-Michel Molina l'étude Anrs Prévenir, qui vise à montrer qu'en déployant la PrEP chez une proportion suffisante des hommes à haut risque (hommes qui ont des relations sexuelles avec les hommes), on observera une baisse de l'incidence de l'infection à VIH dans ce groupe, permettant d'envisager le contrôle de l'épidémie en France.

Une autre des contributions majeures de Dominique Costagliola à la lutte contre l'épidémie d'infections par le VIH est la conduite d'un dispositif, particulièrement innovant à l'époque, d'observation épidémiologique. Après avoir participé en 1988 à la création par Alain-Jacques Valleron de ce qui deviendra la base de données hospitalières française sur l'infection à VIH ANRS CO4 FHDH (French Hospital Database on HIV-ANRS CO4 FHDH), elle en prend la direction en 1992. Alors qu'à cette époque l'idée de mettre en place des cohortes épidémiologiques de longue durée faisait l'objet d'un large scepticisme, cette initiative s'est avérée primordiale dans le domaine de l'étude de l'infection 
à VIH, ayant permis plus de 250 publications de haut niveau. Cette cohorte hospitalière multicentrique où les patients sont inclus en continu, associant 176 hôpitaux, constitue une des plus grandes cohortes de ce type au monde, et participe à plusieurs collaborations internationales. Elle permet, à partir de quelques données biologiques et cliniques structurées, recueillies de façon systématique à l'occasion des soins, de décrire les personnes vivant avec le VIH prises en charge en France et, seule ou en collaboration avec d'autres cohortes des pays du Nord comme du Sud, de faire progresser les connaissances.

Parmi les nombreux travaux auxquels Dominique Costagliola a contribué, on peut également citer l'étude des facteurs pronostiques et du choix du moment de mise en œuvre du traitement, l'étude de l'impact de l'infection à VIH et/ou des traitements sur la morbi-mortalité sévère non-Sida, où, en utilisant les méthodes d'inférence causale en situation observationnelle, elle a notamment montré l'impact de l'immunodépression et de l'activation immune induite par l'infection à VIH sur le risque de cancer, notamment les cancers associés à des virus, et sur le risque d'infarctus. Une de ses contributions importantes en termes de santé publique est d'avoir montré qu'en France, près d'un patient sur trois est pris en charge tardivement et mis ainsi en évidence les conséquences défavorables d'une prise en charge tardive sur le risque de décès.

À partir de 1992, alors que l'arrivée des combinaisons antirétrovirales s'annonce, Dominique Costagliola s'engage également dans le domaine de la recherche clinique en tant que responsable d'un centre de méthodologie, de gestion et d'analyses statistiques de l'Anrs, conduisant de nombreux essais cliniques et des études observationnelles, en particulier avec Christine Katlama, avec laquelle elle travaille notamment sur l'optimisation du traitement antirétroviral. Une de leurs thématiques de recherche récente consiste à questionner, dans le domaine de la prise en charge des patients, des aspects aussi cruciaux que la nécessité d'une trithérapie à vie pour toutes les personnes vivant avec le VIH, ou la possibilité de changer le paradigme d'une trithérapie pour tous par un traitement adapté à chacun, garantissant une charge virale contrôlée.

Outre ces travaux, Dominique Costagliola a une importante activité d'expertise scientifique, en France et à l'étranger, dans différents domaines de santé publique. Elle a aussi joué un rôle très important en matière d'animation et de formation à la recherche, en dirigeant de 2014 à 2018 l'Institut Pierre-Louis d'épidémiologie et de santé publique, dont elle est encore directrice adjointe, et l'École doctorale Pierre-Louis de santé publique à Paris (Sorbonne Université et Université de Paris) de 2011 à 2018.

Aujourd'hui, en ces temps de crise sanitaire, Dominique Costagliola est très active dans la lutte contre la pandémie de COVID-19. Qui ne l'a pas vue à la télévision ou entendu à la radio ? Toujours juste et claire, s'appuyant sur des données établies, elle est, au milieu d'une pléthore d'intervenants dans les médias grand public dont l'expertise est souvent auto-proclamée, une des très rares scientifiques qui sait de quoi elle parle.

Cette brillante carrière, qui mêle des travaux faisant appel aux méthodes complémentaires de l'épidémiologie observationnelle, de la recherche clinique et de la modélisation mathématique, qui a apporté des avancées majeures pour la recherche et pour la santé publique, sans oublier sa profonde implication avec les associations de malades, est largement reconnue, comme notamment le montrent son élection en 2017 à l'Académie des sciences, où elle a rejoint Alain-Jacques Valleron, et le Grand Prix de l'Inserm reçu en 2020. $\diamond$

The 2020 Inserm Grand Prix honoured Dominique Costagliola

\section{LIENS D'INTÉRÊT}

L'auteur déclare n'avoir aucun lien d'intérêt concernant les données publiées dans cet article.

\section{LA FONDATION PREMUP : UN OPÉRATEUR DE TERRAIN EN PÉRINATALITÉ RECONNU POUR SON EXCELLENCE ET SON INTERDISCIPLINARITÉ}

La Fondation de coopération scientifique PremUp, unique en Europe, intervient sur la prévention du handicap à la naissance, par la protection de la santé de la femme enceinte et du nouveau-né.

TIRÉS À PART

M. Goldberg

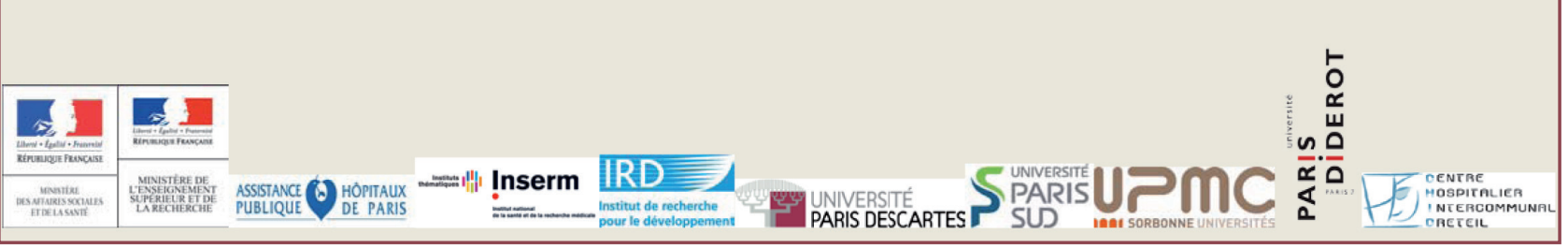

